\title{
Eighth rank isotropic tensors and rotational averages
}

\author{
D L Andrews and W A Ghoul \\ School of Chemical Sciences, University of East Anglia, Norwich NR4 7TJ
}

Received 6 August 1980

\begin{abstract}
Isotropic tensors play an important role in the theory of many physical processes which take place in gases and liquids. In such systems it is usually necessary to perform a rotational average on products of direction cosines relating the space-fixed and molecular coordinate frames. The average is generally expressible in terms of isotropic tensors, and the results for tensors of rank $n \leqslant 7$ have previously been reported. In this paper the isotropic tensors of eighth rank are discussed, and relations between them are demonstrated. The rotational average of eighth rank is then evaluated in both reducible and irreducible form; the results are applicable to a number of processes, for example optical seventh harmonic generation and four-photon absorption.
\end{abstract}

\section{Introduction}

In many areas of physics we encounter systems which are homogeneous on a macroscopic scale. In dealing with the behaviour of such systems under the action of a stimulus with tensor properties, it is necessary to make use of isotropic tensors; these are tensors whose components referred to any Cartesian frame are invariant under rotation of the frame axes. Such tensors play an important role in the microscopic theory of systems with a macroscopic isotropy, for example gases and liquids, where a rotational average is required to account for the random orientations of the component particles.

Consider an isotropic system in which the molecular response to a certain stimulus is given by the scalar product $A_{i_{1} \ldots i_{n}} P_{i_{1} \ldots i_{n}}$, where $A_{i_{1} \ldots i_{n}}$ is a tensor of rank $n$ representing the action of the stimulus, and $P_{i_{1} \ldots i_{n}}$ is a molecular property tensor of the same rank associated with the interaction; here we adopt the implied summation convention for repeated tensor indices. Since the response is in general parametrically dependent upon the molecular orientation with respect to the stimulus, the expression for the corresponding macroscopic observable $T$ is

$$
T=\left\langle A_{i_{1} \ldots i_{n}} P_{i_{1} \ldots i_{n}}\right\rangle,
$$

where the angular brackets denote a rotational average.

If the tensor components in equation (1) are referred to a space-fixed frame, then the values of $P_{i_{1}} \ldots i_{n}$ clearly depend on the molecular orientation, and it is more convenient to refer these tensor components to a molecule-fixed frame in which they are rotation invariant. We therefore write

$$
P_{i_{1} \ldots i_{n}}=l_{i_{1} \lambda_{1}} \ldots l_{i_{n} \lambda_{n}} P_{\lambda_{1} \ldots \lambda_{n}}
$$

where $l_{i_{p} \lambda_{p}}$ is the direction cosine of the angle between the space-fixed axis $i_{p}$ and the 
molecule-fixed axis $\lambda_{p}$. The observable $T$ can then be expressed as

$$
T=A_{i_{1} \ldots i_{n}} P_{\lambda_{1} \ldots \lambda_{n}} I_{i_{1} \ldots i_{n} ; \lambda_{1} \ldots \lambda_{n}}^{(n)}
$$

where $I_{i_{1} \ldots i_{n} ; \lambda_{1} \ldots \lambda_{n}}^{(n)}$ is the rotational average of the direction cosine product $l_{i_{1} \lambda_{1} \ldots i_{n} \lambda_{n}}$. In terms of the Euler angles for the frame transformation, this is given by

$I_{i_{1} \ldots i_{n} ; \lambda_{1} \ldots \lambda_{n}}^{(n)}=\frac{1}{8 \pi^{2}} \int_{0}^{2 \pi} \int_{0}^{\pi} \int_{0}^{2 \pi} l_{i_{1} \lambda_{1}} \ldots l_{i_{n} \lambda_{n}} \sin \theta \mathrm{d} \varphi \mathrm{d} \theta \mathrm{d} \psi$

A systematic method for evaluating rotational averages of this type has been discussed by Andrews and Thirunamachandran (1977), and the result is expressible as a linear combination of products of isotropic tensors referred to the two frames (Weyl 1946). So far only the expressions for tensors of rank $n \leqslant 7$ have been reported, but the higher-order results are increasingly in demand for dealing with nonlinear phenomena. We have found it necessary to evaluate the rotational average of eighth rank in the course of recent studies on four-photon absorption (Andrew and Ghoul 1981); the result is, however, applicable to several other processes, for example optical seventh harmonic generation. In the following section we start with a discussion of the isotropic tensors and relations between them; the eighth-rank rotational average is then presented in $\$ 3$.

\section{Relations between the isotropic tensors}

We start by summarising the properties of isotropic tensors of arbitrary rank $n$. For even $n$, such tensors are products of $n / 2$ Kronecker delta tensors, for example $\delta_{i_{1} i_{2}} \delta_{i_{3} i_{4}} \ldots \delta_{i_{n-1} i_{n}}$, whereas for odd $n$ they are products of one Levi-Civita antisymmetric tensor and $(n-3) / 2$ Kronecker deltas, for example $\varepsilon_{i_{1} i_{2} i_{3}} \delta_{i_{4} i_{5}} \ldots \delta_{i_{n-1} i_{n}}$. In each case there are other tensors of isomeric form which can be cbtained by index permutation; the full number is given by

$$
\begin{aligned}
& N_{n}=\frac{n !}{2^{n / 2}(n / 2) !}, \quad(n \text { even }) \\
& N_{n}=\frac{n !}{3.2^{(n-1) / 2}((n-3) / 2) !}, \quad(n \text { odd }) .
\end{aligned}
$$

This set of tensors is in general overcomplete, however, and the number of linearly independent tensors is given by

$$
Q_{n}=\sum_{r=0}^{p} \frac{n !(3 r-n+1)}{(n-2 r) ! r !(r+1) !}
$$

where $p$ assumes the value [n/2], i.e. the integer part of $n / 2$. The number of isomers $N_{n}$ and the size of the linearly independent set $Q_{n}$ are shown in table 1 for $n=2$ to 10 ; we find that $Q_{n}<N_{n}$ for odd $n \geqslant 5$ and for even $n \geqslant 8$. The procedure for choosing a suitable complete and linearly independent subset of $Q_{n}$ tensors from the set of $N_{n}$ isomers has been established by Smith (1968), and involves the construction of standard tableaux from Young diagrams.

Consider the partition of $n$ given by the sequence of integers $\left(n_{1}, n_{2} \ldots, n_{r}\right)$ whose sum is $n$, with $n_{1} \geqslant n_{2} \ldots \geqslant n_{r}$. Corresponding to each partition we construct a frame of 
Table 1. The number of isotropic tensor isomers $N_{n}$ and the size $Q_{n}$ of the linearly independent basis set for rank $n$.

\begin{tabular}{|c|c|c|c|c|c|c|c|c|c|}
\hline$n$ & 2 & 3 & 4 & 5 & 6 & 7 & 8 & 9 & 10 \\
\hline$N_{n}$ & 1 & 1 & 3 & 10 & 15 & 105 & 105 & 1260 & 945 \\
\hline$Q_{n}$ & 1 & 1 & 3 & 6 & 15 & 36 & 91 & 232 & 603 \\
\hline
\end{tabular}

$n$ squares in rows and columns, with $n_{i}$ squares in row $i$, the first elements in each row lying directly above one another. For the present application it is necessary to restrict $n_{\text {i }}$ to even values, and the relevant frames for $n=8$ are therefore those corresponding to the partitions $(8) ;(6,2) ;(4,4) ;(4,2,2)$ and $(2,2,2,2)$ as shown in figure 1.
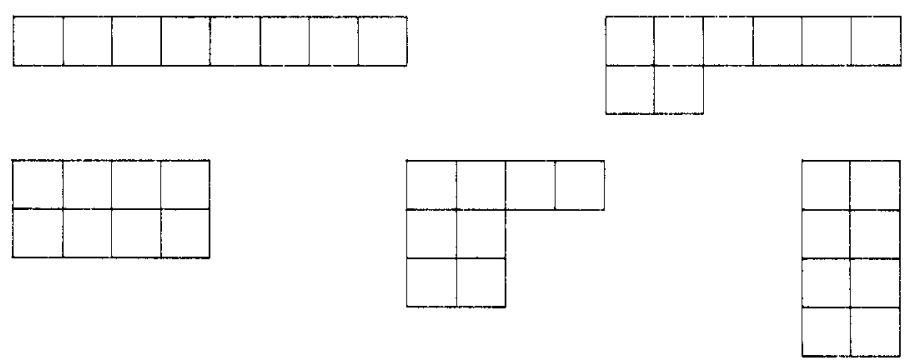

Figure 1. Frames for the eighth-rank isotropic tensors.

Standard tableaux are obtained from each frame by inserting the numbers 1 to $n$ in such a way that they increase in every row from left to right, and in every column reading downwards. The number of standard tableaux belonging to the frame $\left(n_{1}, n_{2} \ldots, n_{r}\right)$ is equal to the degree of the irreducible representation $d_{n_{1}, n_{2} \ldots, n_{r}}$, and is given by (Smith 1968);

$$
d_{n_{1}, n_{2} \ldots, n_{r}}=n ! \prod_{j<k}\left(a_{j}-a_{k}\right) / a_{1} ! a_{2} ! \ldots a_{r} !
$$

where

$$
a_{j}=n_{j}+r-j
$$

It is possible to identify with each standard tableau, a linear combination of isotropic tensors, by constructing a generalised Kronecker delta tensor (Sokolnikoff 1964) from each pair of columns. If the entries in one column are $\alpha$ to $\beta$, and those in the adjacent column are $\gamma$ to $\delta$, the appropriate tensor is $\delta_{i_{\gamma} \ldots i_{\delta}}^{i_{\alpha} \ldots i_{\beta}}$, defined by

$$
\delta_{i_{\gamma} \ldots i_{\delta}}^{i_{\alpha} \ldots i_{\beta}}=\left|\begin{array}{ccc}
\delta_{i_{\alpha} i_{\gamma}} & \ldots & \delta_{i_{\alpha} i_{\delta}} \\
\vdots & & \vdots \\
\delta_{i_{\beta} i_{\gamma}} & \ldots & \delta_{i_{\beta} i_{\delta}}
\end{array}\right| .
$$

For example, the standard tableau of figure 2(a) is associated with the tensor $\delta_{i_{2} i_{8}}^{i_{1} i_{7}} \delta_{i_{4}}^{i_{3}} \delta_{i_{6}}^{i_{5}}=\delta_{i_{1} i_{2}} \delta_{i_{7} i_{8}} \delta_{i_{3} i_{4}} \delta_{i_{5} i_{6}}-\delta_{i_{1} i_{8}} \delta_{i_{2} i_{7}} \delta_{i_{3} i_{4}} \delta_{i_{5} i_{6}}$.

However, tensors corresponding to standard tableaux with a greater number of rows than the dimensionality are null tensors (Smith 1968, 1972). Consequently in threedimensional space each linear combination of tensors constructed from a standard 


\begin{tabular}{|l|l|l|l|l|l|}
\hline 1 & 2 & 3 & 4 & 5 & 6 \\
\hline 7 & 8 & & & \\
\cline { 1 - 2 } & &
\end{tabular}

\begin{tabular}{|l|l|}
\hline 1 & 5 \\
\hline 2 & 6 \\
\hline 3 & 7 \\
\hline 4 & 8 \\
\hline
\end{tabular}

Figure 2. Examples of the standard tableaux corresponding to eighth-rank isotropic tensors.

tableau of frame $(2,2,2,2)$ is identically zero; from equation (8) we find that there are fourteen such tableaux. Writing in full the linear combination corresponding to the standard tableau of figure $2(b)$, for example, we thus obtain the following relation between eighth-rank isotropic tensors (Rivlin 1955, Kearsley and Fong 1975);

$$
\left|\begin{array}{llll}
\delta_{i_{1} i_{5}} & \delta_{i_{1} i_{6}} & \delta_{i_{1} i_{7}} & \delta_{i_{1} i_{8}} \\
\delta_{i_{2} i_{5}} & \delta_{i_{2} i_{6}} & \delta_{i_{2} i_{7}} & \delta_{i_{2} i_{8}} \\
\delta_{i_{3} i_{5}} & \delta_{i_{3} i_{6}} & \delta_{i_{3} i_{7}} & \delta_{i_{3} i_{8}} \\
\delta_{i_{4} i_{5}} & \delta_{i_{4} i_{6}} & \delta_{i_{4} i_{7}} & \delta_{i_{4} i_{8}}
\end{array}\right|=0
$$

An interesting identity concerning scalar products of vectors follows from this equation, and is presented in an Appendix. There are thirteen other relations of similar form to equation (11), each corresponding to a particular standard tableau, and obtainable from this equation by index permutation. These relations enable us to choose a suitable basis set of 91 linearly independent isotropic tensors, in terms of which the remaining 14 isomers can be expressed.

Before proceeding further, it is worth noting that from equation (11) there follow several further identities concerning isotropic tensors of other ranks where the set of tensor isomers is overcomplete. First, by taking the inner product with the Levi-Civita tensor $\varepsilon_{i_{6} i_{7} i_{8}}$ we recover a well known relation between fifth-rank isotropic tensors (Caldonazzo 1932, Boyle and Matthews 1971 and Healy 1975);

$$
\varepsilon_{i_{2} i_{3} i_{4}} \delta_{i_{1} i_{5}}=\varepsilon_{i_{1} i_{2} i_{3}} \delta_{i_{4} i_{5}}-\varepsilon_{i_{1} i_{2} i_{4}} \delta_{i_{3} i_{5}}+\varepsilon_{i_{1} i_{3} i_{4}} \delta_{i_{2} i_{5}}
$$

This relation, together with three others obtained by index permutation, allows the overcomplete set of 10 fifth-rank tensors to be reduced to 6 . Conversely, the eighthrank identities can be derived from the fifth-rank relations by taking their outer product with $\varepsilon_{i_{6} i 7 i_{8}}$ and making use of the formula

$$
\varepsilon_{i_{a} i_{b} i_{c}} \varepsilon_{i_{d} i_{e} i_{f}}=\left|\begin{array}{lll}
\delta_{i_{a} i_{d}} & \delta_{i_{a} i_{e}} & \delta_{i_{a} i_{f}} \\
\delta_{i_{b} i_{d}} & \delta_{i_{b} i_{e}} & \delta_{i_{b} i_{f}} \\
\delta_{i_{c} i_{d}} & \delta_{i_{c} i_{e}} & \delta_{i_{c} i_{f}}
\end{array}\right| .
$$

Similarly, we can derive relations between seventh-rank isotropic tensors by contracting equation (11) and its index permutations with a Levi-Civita tensor having just two indices from the set $i_{1} \ldots i_{8}$. For example contraction with $\varepsilon_{i_{7} i_{8} i_{9}}$ gives;

$$
\begin{aligned}
\varepsilon_{i_{1} i_{2} i_{7}} \delta_{i_{3} i_{5}} \delta_{i_{4} i_{6}} & -\varepsilon_{i_{1} i_{2} i_{7}} \delta_{i_{3} i_{6}} \delta_{i_{4} i_{5}}-\varepsilon_{i_{1} i_{3} i_{7}} \delta_{i_{2} i_{5}} \delta_{i_{4} i_{6}}+\varepsilon_{i_{1} i_{3} i_{7}} \delta_{i_{2} i_{6}} \delta_{i_{4} i_{5}} \\
& +\varepsilon_{i_{1} i_{4} i_{7}} \delta_{i_{2} i_{5}} \delta_{i_{3} i_{6}}-\varepsilon_{i_{1} i_{4} i_{7}} \delta_{i_{2} i_{6}} \delta_{i_{3} i_{5}}+\varepsilon_{i_{2} i_{3} i_{7}} \delta_{i_{1} i_{5}} \delta_{i_{4} i_{6}}-\varepsilon_{i_{2} i_{3} i_{7}} \delta_{i_{1} i_{6}} \delta_{i_{4} i_{5}} \\
& -\varepsilon_{i_{2} i_{4} i_{7}} \delta_{i_{1} i_{5}} \delta_{i_{3} i_{6}}+\varepsilon_{i_{2} i_{4} i_{7}} \delta_{i_{1} i_{6}} \delta_{i_{3} i_{5}}+\varepsilon_{i_{3} i_{4} i_{7}} \delta_{i_{1} i_{5}} \delta_{i_{2} i_{6}}-\varepsilon_{i_{3} i_{4} i_{7}} \delta_{i_{1} i_{6}} \delta_{i_{2} i_{5}}=0,
\end{aligned}
$$


following the substitution $i_{9} \rightarrow i_{7}$. In all, there are 69 independent relations between seventh-rank isotropic tensors, which can be used to reduce the overcomplete set of 105 tensors to a linearly independent set of 36 ; these relations have previously been obtained by Andrews (1976).

Finally, these methods can be extended to provide important new relations between isotropic tensors of rank $n>8$, simply by taking the product of equations (11) and (14) with appropriate Kronecker delta or Levi-Civita tensors. Such identities will prove valuable in the calculation of higher rank rotational averages.

\section{The eighth-rank rotational average}

As stated earlier, the rotational average defined by equation (4) is expressible as a linear combination of products of isotropic tensors referred to the space-fixed and moleculefixed frames. We shall now denote eighth-rank isotropic tensors referred to the space-fixed frame by $f_{i_{1}}^{(r)} \ldots i_{8}$, and the corresponding tensors referred to the moleculefixed frame by $g_{\lambda_{1} \ldots \lambda_{8}}^{(r)}$; the explicit form of the linearly independent set of 91 tensor isomers is given by table 2 , in which

$$
\begin{aligned}
& f_{i_{1} \ldots i_{s}}^{(r)}=\delta_{i_{a} i_{b}} \delta_{i_{c} i_{d}} \delta_{i_{e} i_{f}} \delta_{i_{g} i_{h}} ; \\
& g_{\lambda_{1} \ldots \lambda_{s}}^{(r)}=\delta_{\lambda_{a} \lambda_{b}} \delta_{\lambda_{c} \lambda_{d}} \delta_{\lambda_{g} \lambda_{f}} \delta_{\lambda_{g} \lambda_{h} .} .
\end{aligned}
$$

Thus in general we can write

$$
I_{i_{1} \ldots i_{8} ; \lambda_{1} \ldots \lambda_{8}}^{(8)}=\sum_{r, s=1}^{91} m_{r s}^{(8)} f_{i_{1} \ldots i_{8}}^{(r)} g_{\lambda_{1} \ldots \lambda_{8}}^{(s)},
$$

where the coefficients $m_{r s}^{(8)}$ are real numbers. As shown previously (Andrews and Thirunamachandran 1977), the matrix $M^{(8)}$ whose elements are these coefficients can be obtained by a straightforward matrix inversion procedure; we find

$$
M^{(8)}=\left(S^{(8)}\right)^{-1}
$$

where the elements $S_{r s}^{(8)}$ of $S^{(8)}$ are calculated from the tensor inner product

$$
s_{r s}^{(8)}=f_{i_{1} \ldots i_{8}}^{(r)} f_{i_{1} \ldots i_{8}}^{(s)}
$$

We have calculated the matrix $M^{(8)} \dagger$, and hence the full result for $I_{i_{1}}^{(8)} \ldots i_{8} ; \lambda_{1} \ldots \lambda_{8}$ using this method, but the resultant expression is awkward since $M^{(8)}$ has no discernible structure apart from being symmetric, and the tensor basis set is not particularly convenient for practical applications.

We have therefore derived from this result an alternative, yet completely equivalent expression for the eighth-rank rotational average, by employing the overcomplete set of 105 tensor isomers as basis. For this purpose equation (17) is rewritten as follows;

$$
I_{i_{1} \ldots i_{8} ; \lambda_{1} \ldots \lambda_{8}}^{(8)}=\sum_{r^{\prime}, s^{\prime}=1}^{105} m_{r^{\prime} s^{\prime}}^{(8)} f_{i_{1} \ldots i_{8}}^{\prime\left(r^{\prime}\right)} g_{\lambda_{1} \ldots \lambda_{8}}^{\prime\left(s^{\prime}\right)}
$$

$\dagger$ A copy of the matrix $M^{(8)}$ is held by the British Library Supplementary Publications Scheme and is available from the British Library Lending Division, Boston Spa, Wetherby, Yorks, LS23 7 QB (reference SUP 70030). 
Table 2. The complete and overcomplete sets of eighth-rank isotropic tensors defined by equations (15) and (21).

\begin{tabular}{|c|c|c|c|c|c|c|c|c|c|c|c|c|c|c|c|c|c|}
\hline$r$ & $r^{\prime}$ & $a b$ & $c d$ & $e f$ & $g h$ & $r$ & $r^{\prime}$ & $a b$ & $c d$ & $e f$ & $g h$ & $r$ & $r^{\prime}$ & $a b$ & $c d$ & $e f$ & $g h$ \\
\hline- & 1 & 12 & 34 & 56 & 78 & 24 & 36 & 14 & 25 & 38 & 67 & 57 & 71 & 16 & 27 & 35 & 48 \\
\hline- & 2 & 12 & 34 & 57 & 68 & 25 & 37 & 14 & 26 & 35 & 78 & 58 & 72 & 16 & 27 & 38 & 45 \\
\hline 1 & 3 & 12 & 34 & 58 & 67 & - & 38 & 14 & 26 & 37 & 58 & 59 & 73 & 16 & 28 & 34 & 57 \\
\hline- & 4 & 12 & 35 & 46 & 78 & 26 & 39 & 14 & 26 & 38 & 57 & 60 & 74 & 16 & 28 & 35 & 47 \\
\hline- & 5 & 12 & 35 & 47 & 68 & 27 & 40 & 14 & 27 & 35 & 68 & 61 & 75 & 16 & 28 & 37 & 45 \\
\hline 2 & 6 & 12 & 35 & 48 & 67 & 28 & 41 & 14 & 27 & 36 & 58 & 62 & 76 & 17 & 23 & 45 & 68 \\
\hline 3 & 7 & 12 & 36 & 45 & 78 & 29 & 42 & 14 & 27 & 38 & 56 & 63 & 77 & 17 & 23 & 46 & 58 \\
\hline- & 8 & 12 & 36 & 47 & 58 & 30 & 43 & 14 & 28 & 35 & 67 & 64 & 78 & 17 & 23 & 48 & 56 \\
\hline 4 & 9 & 12 & 36 & 48 & 57 & 31 & 44 & 14 & 28 & 36 & 57 & 65 & 79 & 17 & 24 & 35 & 68 \\
\hline 5 & 10 & 12 & 37 & 45 & 68 & 32 & 45 & 14 & 28 & 37 & 56 & 66 & 80 & 17 & 24 & 36 & 58 \\
\hline 6 & 11 & 12 & 37 & 46 & 58 & 33 & 46 & 15 & 23 & 46 & 78 & 67 & 81 & 17 & 24 & 38 & 56 \\
\hline 7 & 12 & 12 & 37 & 48 & 56 & 34 & 47 & 15 & 23 & 47 & 68 & 68 & 82 & 17 & 25 & 34 & 68 \\
\hline 8 & 13 & 12 & 38 & 45 & 67 & 35 & 48 & 15 & 23 & 48 & 67 & 69 & 83 & 17 & 25 & 36 & 48 \\
\hline 9 & 14 & 12 & 38 & 46 & 57 & 36 & 49 & 15 & 24 & 36 & 78 & 70 & 84 & 17 & 25 & 38 & 46 \\
\hline 10 & 15 & 12 & 38 & 47 & 56 & 37 & 50 & 15 & 24 & 37 & 68 & 71 & 85 & 17 & 26 & 34 & 58 \\
\hline- & 16 & 13 & 24 & 56 & 78 & 38 & 51 & 15 & 24 & 38 & 67 & 72 & 86 & 17 & 26 & 35 & 48 \\
\hline- & 17 & 13 & 24 & 57 & 68 & 39 & 52 & 15 & 26 & 34 & 78 & 73 & 87 & 17 & 26 & 38 & 45 \\
\hline 11 & 18 & 13 & 24 & 58 & 67 & $\ldots$ & 53 & 15 & 26 & 37 & 48 & 74 & 88 & 17 & 28 & 34 & 56 \\
\hline- & 19 & 13 & 25 & 46 & 78 & 40 & 54 & 15 & 26 & 38 & 47 & 75 & 89 & 17 & 28 & 35 & 46 \\
\hline- & 20 & 13 & 25 & 47 & 68 & 41 & 55 & 15 & 27 & 34 & 68 & 76 & 90 & 17 & 28 & 36 & 45 \\
\hline 12 & 21 & 13 & 25 & 48 & 67 & 42 & 56 & 15 & 27 & 36 & 48 & 77 & 91 & 18 & 23 & 45 & 67 \\
\hline 13 & 22 & 13 & 26 & 45 & 78 & 43 & 57 & 15 & 27 & 38 & 46 & 78 & 92 & 18 & 23 & 46 & 57 \\
\hline- & 23 & 13 & 26 & 47 & 58 & 44 & 58 & 15 & 28 & 34 & 67 & 79 & 93 & 18 & 23 & 47 & 56 \\
\hline 14 & 24 & 13 & 26 & 48 & 57 & 45 & 59 & 15 & 28 & 36 & 47 & 80 & 94 & 18 & 24 & 35 & 67 \\
\hline 15 & 25 & 13 & 27 & 45 & 68 & 46 & 60 & 15 & 28 & 37 & 46 & 81 & 95 & 18 & 24 & 36 & 57 \\
\hline 16 & 26 & 13 & 27 & 46 & 58 & 47 & 61 & 16 & 23 & 45 & 78 & 82 & 96 & 18 & 24 & 37 & 56 \\
\hline 17 & 27 & 13 & 27 & 48 & 56 & 48 & 62 & 16 & 23 & 47 & 58 & 83 & 97 & 18 & 25 & 34 & 67 \\
\hline 18 & 28 & 13 & 28 & 45 & 67 & 49 & 63 & 16 & 23 & 48 & 57 & 84 & 98 & 18 & 25 & 36 & 47 \\
\hline 19 & 29 & 13 & 28 & 46 & 57 & 50 & 64 & 16 & 24 & 35 & 78 & 85 & 99 & 18 & 25 & 37 & 46 \\
\hline 20 & 30 & 13 & 28 & 47 & 56 & 51 & 65 & 16 & 24 & 37 & 58 & 86 & 100 & 18 & 26 & 34 & 57 \\
\hline 21 & 31 & 14 & 23 & 56 & 78 & 52 & 66 & 16 & 24 & 38 & 57 & 87 & 101 & 18 & 26 & 35 & 47 \\
\hline 22 & 32 & 14 & 23 & 57 & 68 & 53 & 67 & 16 & 25 & 34 & 78 & 88 & 102 & 18 & 26 & 37 & 45 \\
\hline 23 & 33 & 14 & 23 & 58 & 67 & 54 & 68 & 16 & 25 & 37 & 48 & 89 & 103 & 18 & 27 & 34 & 56 \\
\hline- & 34 & 14 & 25 & 36 & 78 & 55 & 69 & 16 & 25 & 38 & 47 & 90 & 104 & 18 & 27 & 35 & 46 \\
\hline - & 35 & 14 & 25 & 37 & 68 & 56 & 70 & 16 & 27 & 34 & 58 & 91 & 105 & 18 & 27 & 36 & 45 \\
\hline
\end{tabular}

where $f_{i_{1} \ldots i_{8}}^{\prime\left(r^{\prime}\right)}$ and its molecular-frame counterpart are again given by table 2 . We shall refer to this form of the rotational average as reducible, in distinction from the irreducible result which is expressed in terms of the linearly independent basis set. Because of the overcompleteness of the basis in the reducible case, the coefficients in equation (20) are not uniquely defined, in contrast to the $m_{r s}^{(8)}$ in equation (17), and thus we have the freedom to impose certain conditions on the structural form of the matrix $M^{\prime(8)}$ whose elements are $m_{r^{\prime} s^{\prime}}^{\prime(8)}$. A similar situation arises in the case of the fifth-and seventh-rank rotational averages, which have been dealt with previously (Andrews and Thirunamachandran 1977).

We have found it most convenient to impose on $M^{(8)}$ the same structure as the matrix $S^{\prime(8)}$, which is the analogue of $S^{(8)}$ with the overcomplete set of 105 tensors as basis. Here there are only five distinct types of matrix element, which we represent by the variables $A, B, C, D$ and $E$. The variable assigned to any particular matrix element 
can be determined by the following simple method. Consider the matrix element $m_{r^{\prime} s^{\prime}}^{\prime(8)}$, where

$$
\begin{aligned}
& f_{i_{1} \ldots i_{8}}^{\left(r^{\prime}\right)}=\delta_{i_{a} i_{b}} \delta_{i_{c} i_{d}} \delta_{i_{e} i_{f}} \delta_{i_{g} i_{h}} ; \\
& f_{i_{1} \ldots i_{8}}^{\prime\left(s^{\prime}\right)}=\delta_{i_{i} i_{i}} \delta_{i_{k} i_{l}} \delta_{i_{m} i_{n}} \delta_{i_{o} i_{p}},
\end{aligned}
$$

and the values of $a \ldots h, i \ldots p$ are integers in the range $1-8$, given by table 2 . Each tensor can be represented by four index pairs, i.e. $(a b)(c d)(e f)(g h)$ and $(i j)(k l)(m n)(o p)$, and together these link the indices in one or more closed cycles. For example for $m_{1,105}^{\prime(8)}$ we have the index pairs $(12)(34)(56)(78)$ and $(18)(27)(36)(45)$ giving cycles $1 \rightarrow 2 \rightarrow 7 \rightarrow$ $8 \rightarrow 1$ and $3 \rightarrow 4 \rightarrow 5 \rightarrow 6 \rightarrow 3$, which we can write as (1278)(3456). The way in which the eight indices are thereby divided into cycles determines which of the variables $A-E$ we assign to the matrix eiement; there are five possibilities corresponding to the even partitions of 8 , i.e. $(2,2,2,2) ;(4,2,2) ;(4,4) ;(6,2) ;(8)$. In the example given, (1278)(3456) corresponds to the partition $(4,4)$, i.e. two cycles each containing four integers. The value of the matrix element associated with each type of partition is given in table 3 , together with examples of the corresponding cycle structures.

Table 3. Assignment of the variables $A-E$ to elements of the matrix $M^{\prime(8)}$.

\begin{tabular}{lll}
\hline Example & Partition & $\begin{array}{l}\text { Matrix } \\
\text { element }\end{array}$ \\
\hline$(12)(34)(56)(78) \otimes(12)(34)(56)(78)=(12)(34)(56)(78)$ & $(2,2,2,2)$ & $A$ \\
$(12)(34)(56)(78) \otimes(13)(24)(56)(78)=(1243)(56)(78)$ & $(4,2,2)$ & $B$ \\
$(12)(34)(56)(78) \otimes(13)(24)(57)(68)=(1243)(5687)$ & $(4,4)$ & $C$ \\
$(12)(34)(56)(78) \otimes(13)(25)(46)(78)=(125643)(78)$ & $(6,2)$ & $D$ \\
$(i 2)(34)(56)(78) \otimes(13)(25)(47)(68)=(12568743)$ & $(8)$ & $E$ \\
\hline
\end{tabular}

Using this method it is a quite straightforward matter to generate the $105 \times$ $105 M^{\prime 8)}$ matrix using a computer; the result is given in table 4 . It then remains to find values for the variables $A-E$. This can be achieved by reducing $M^{\prime(8)}$ to irreducible form using the relations which express the 14 redundant tensors in terms of the linearly independent set; the relevant equations are obtainable from the fourteen index permutations of equation (11). We can then construct a $105 \times 91$ matrix $H \dagger$, whose elements are the coefficients in the linear combinations which relate the overcomplete set to the linearly independent set;

$$
\begin{aligned}
& f_{i_{1} \ldots i_{8}}^{\left(r^{\prime}\right)}=\sum_{r=1}^{91} h_{r^{\prime} r} f_{i_{1}}^{(r)} \ldots i_{8} ; \\
& g_{\lambda_{1} \ldots \lambda_{8}}^{\prime\left(s^{\prime}\right)}=\sum_{s=1}^{91} h_{s^{\prime} s} g_{\lambda_{1} \ldots \lambda_{8} .}^{(s)} .
\end{aligned}
$$

The reducible $105 \times 105$ matrix $M^{\prime(8)}$ is then related to the irreducible $91 \times 91$ matrix $M^{(8)}$ through the relation

$$
M^{(8)}=H^{T} M^{(8)} H
$$

\footnotetext{
+ A copy of the matrix $H$ is held by the British Library Supplementary Publications Scheme and is available from the British Library Lending Division, Boston Spa, Wetherby, Yorks, LS23 7QB (reference SUP 70030).
} 
Table 4. The reducible form of the eighth-rank rotational averaging matrix $M^{\prime(8)}$.

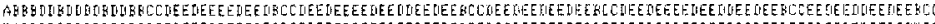

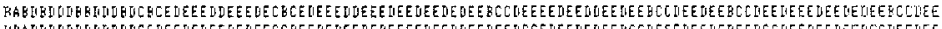

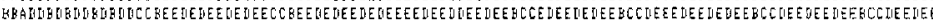

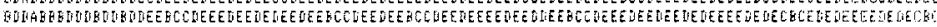

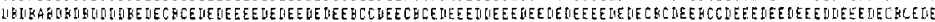

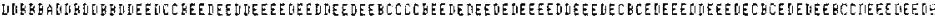

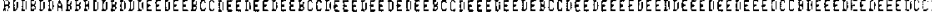

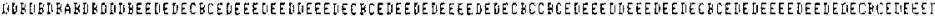

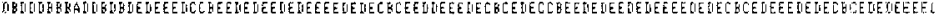

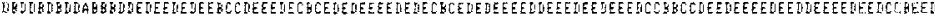

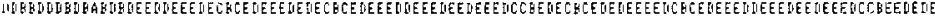

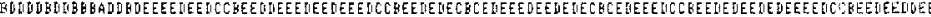

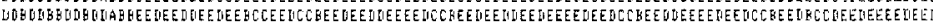

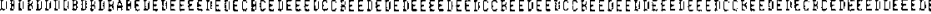

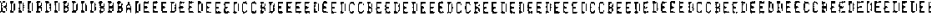

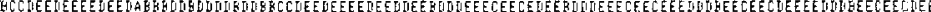

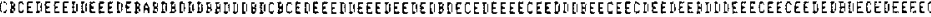

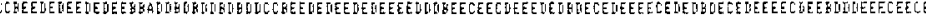

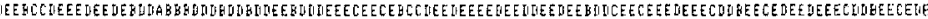

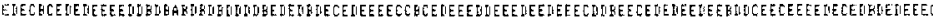

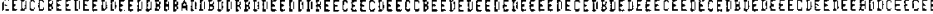

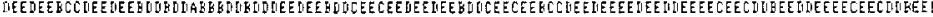
-EDEUECHCE DEEEDUDEE

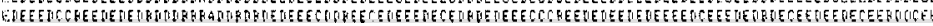

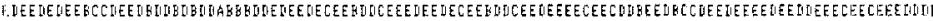

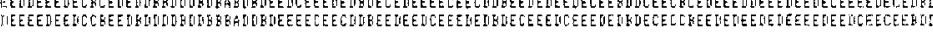

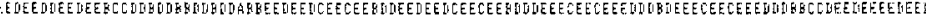

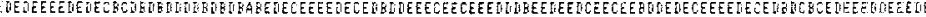

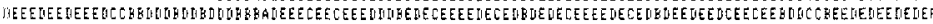

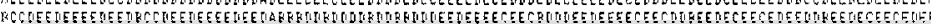

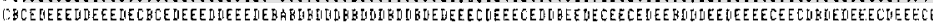

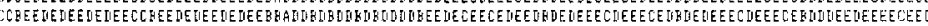

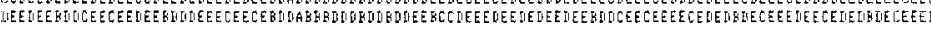

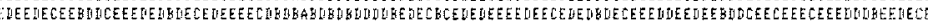

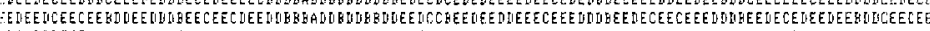

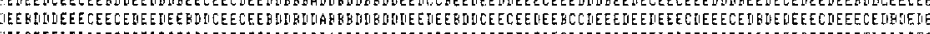

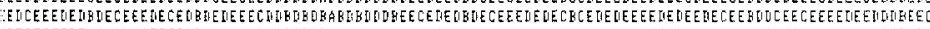

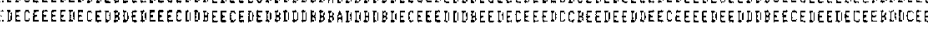

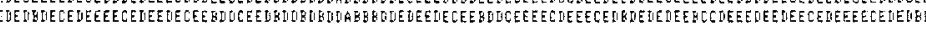

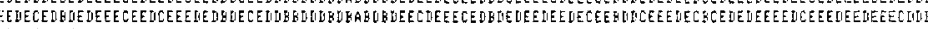

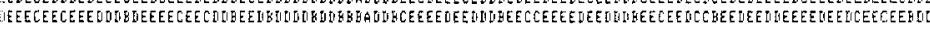

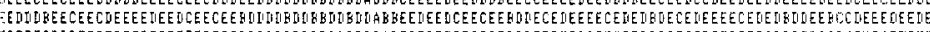

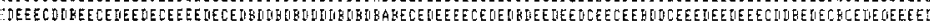

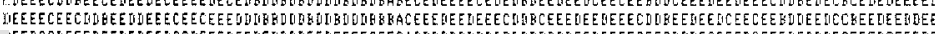

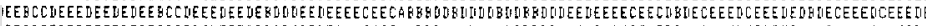

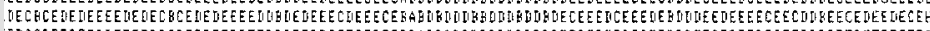

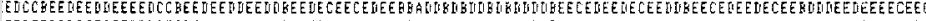

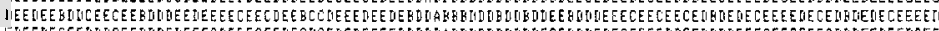

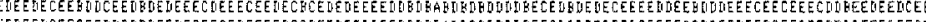

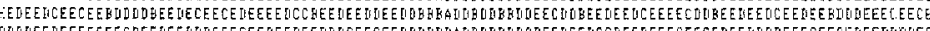

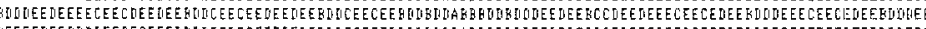

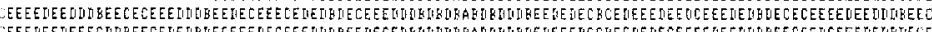

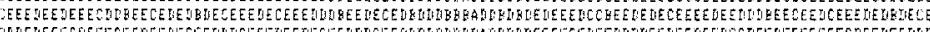

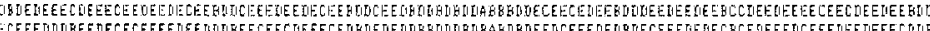

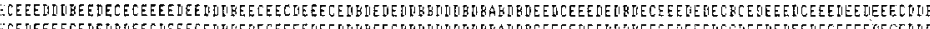

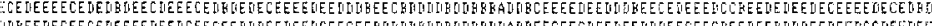

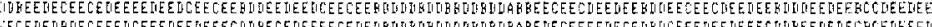

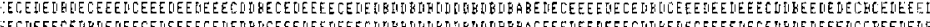

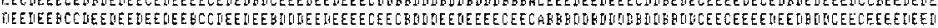

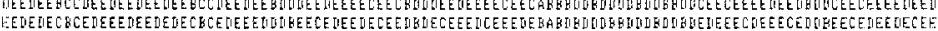

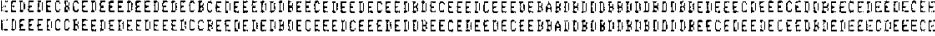

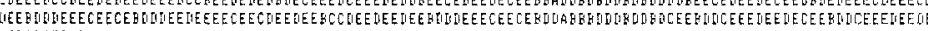

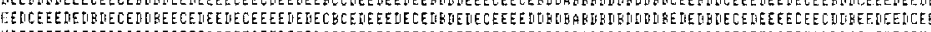

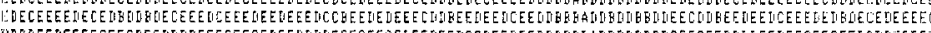

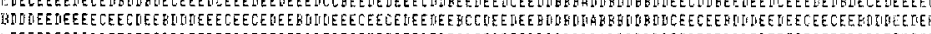

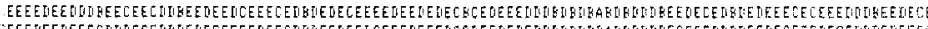
CeEe

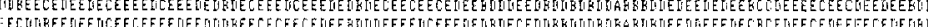

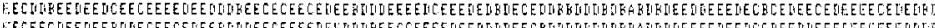

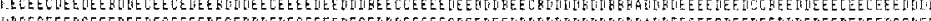
 CEDSDEACCEeC

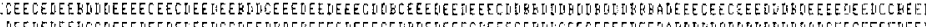

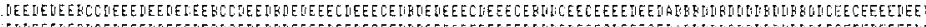

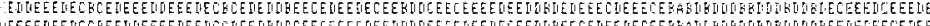

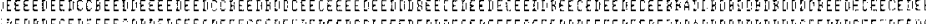

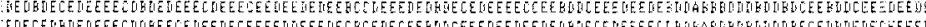

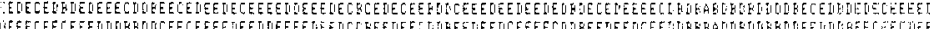

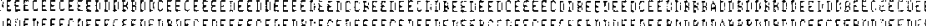
B

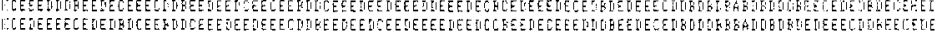

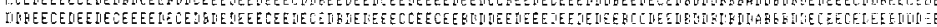
WECTIB EETEE TCEEECEE

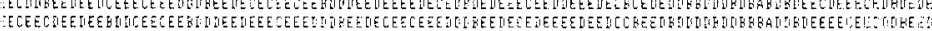
HIDCEE

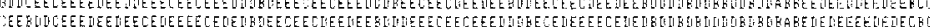

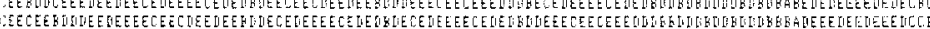
ECEEOT OLESCCE TELEE EIEUECOC

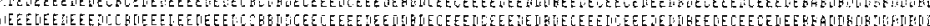

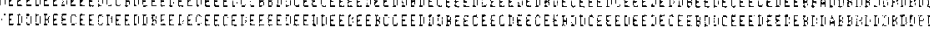

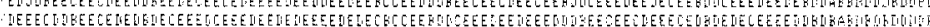

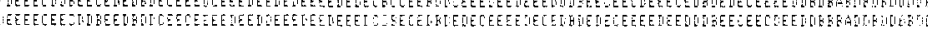

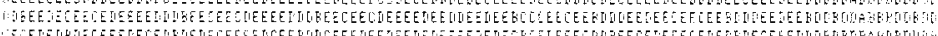

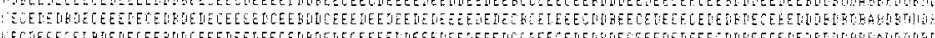

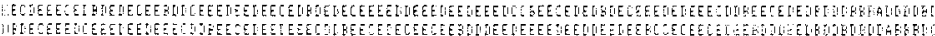
WE

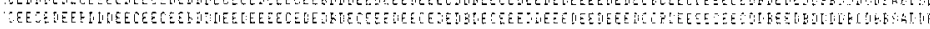

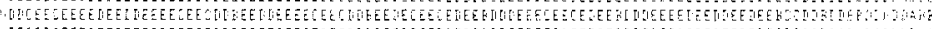

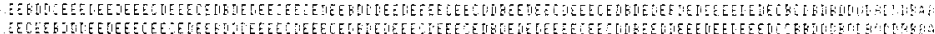


By comparing the right-hand side of equation (25) with the result for $M^{(8)}$ obtained previously, we obtain four independent equations in terms of the variables $A-E$;

$$
\begin{aligned}
& A+2 B=17 / 945 \\
& B+2 C=-11 / 1890 \\
& B+2 D=-13 / 3780 \\
& D+2 E=1 / 756
\end{aligned}
$$

Any set of coefficients which satisfies these equations can be substituted in $M^{\prime(8)}$ and the result used in equation (20) as a reducible form of the eighth-rank rotational average. We have the freedom to choose the value of any one variable, and we have found it most convenient to set the most frequently occurring matrix element $E$ to zero, leading to the following values for the other parameters; $A=228 / 7560 ; B=-46 / 7560 ; C=$ $1 / 7560 ; D=10 / 7560$. It is important to note that the reducible form of the rotational average can be used directly, without reduction to irreducible form.

Using formulae given previously (Andrews and Thirunamachandran 1977), we have verified that both the reducible and irreducible forms of the eighth-rank rotational average correctly reduce to the known results for the rotational averages of lower rank. Finally, we have also established that the relations

$$
\sum_{r, s=1}^{91} m_{r s}^{(8)}=\sum_{r, s=1}^{105} m_{r s}^{\prime(8)}=\frac{1}{9}
$$

hold, in accordance with the general result (Andrews 1980)

$$
\sum_{r, s=1}^{O_{2 n}} m_{r s}^{(2 n)}=\sum_{r, s=1}^{N_{2 n}} m_{r s}^{\prime(2 n)}=\frac{1}{2 n+1}
$$

\section{Acknowledgment}

We would like to express our thanks to Dr T Thirunamachandran for most helpful suggestions on this work. One of us (WAG) also gratefully acknowledges financial support from the Lebanese University.

\section{Appendix. A scalar product identity}

In certain physical problems the eighth-rank molecular response tensor $P_{\lambda_{1}} \ldots \lambda_{8}$ is expressible as a product of vector components $a_{\lambda_{1}} b_{\lambda_{2}} c_{\lambda_{3}} d_{\lambda_{4}} a_{\lambda_{5}} b_{\lambda_{6}} c_{\lambda_{7}} d_{\lambda_{8}}$. The result for the observable $T$ given by equation (3) of the main text then involves parameters of the form $a_{\lambda_{1}} b_{\lambda_{2}} c_{\lambda_{3}} d_{\lambda_{4}} a_{\lambda_{s}} b_{\lambda_{6}} c_{\lambda_{7}} d_{\lambda_{8}} g_{\lambda_{1}}^{(s)} \ldots \lambda_{8}$. Since $g_{\lambda_{1} \ldots \lambda_{8}}^{(s)}$ is a product of four Kronecker deltas, these parameters consist of four scalar products formed from four pairs of identical vectors. The number of distinct parameters of this kind is given by the coefficient of $x^{4}$ in the expansion of $(1-x)^{-1 / 2} \exp \left(x / 2+x^{2} / 4\right)$ (Cayley 1874), which is 17. From equation (11), it follows that there is a unique linear relationship between 
these parameters, which can be written most simply in terms of the unit vectors as follows;

$$
\begin{aligned}
(\hat{a} \cdot \hat{b})^{2}+(\hat{a} \cdot \hat{c})^{2} & +(\hat{a} \cdot \hat{d})^{2}+(\hat{b} \cdot \hat{c})^{2}+(\hat{b} \cdot \hat{d})^{2} \\
& +(\hat{c} \cdot \hat{d})^{2}-(\hat{a} \cdot \hat{b})^{2}(\hat{c} \cdot \hat{d})^{2}-(\hat{a} \cdot \hat{c})^{2}(\hat{b} \cdot \hat{d})^{2} \\
& -(\hat{a} \cdot \hat{d})^{2}(\hat{b} \cdot \hat{c})^{2}-2(\hat{a} \cdot \hat{b})(\hat{b} \cdot \hat{c})(\hat{c} \cdot \hat{a})-2(\hat{a} \cdot \hat{b})(\hat{b} \cdot \hat{d})(\hat{d} \cdot \hat{a}) \\
& -2(\hat{a} \cdot \hat{c})(\hat{c} \cdot \hat{d})(\hat{d} \cdot \hat{a})-2(\hat{b} \cdot \hat{c})(\hat{c} \cdot \hat{d})(\hat{d} \cdot \hat{b})+2(\hat{a} \cdot \hat{b})(\hat{b} \cdot \hat{c})(\hat{c} \cdot \hat{d})(\hat{d} \cdot \hat{a}) \\
& +2(\hat{a} \cdot \hat{b})(\hat{b} \cdot \hat{d})(\hat{d} \cdot \hat{c})(\hat{c} \cdot \hat{a})+2(\hat{a} \cdot \hat{c})(\hat{c} \cdot \hat{b})(\hat{b} \cdot \hat{d})(\hat{d} \cdot \hat{a})=1 .
\end{aligned}
$$

This is a perfectly general result for any real vectors $\boldsymbol{a}, \boldsymbol{b}, \boldsymbol{c}$ and $\boldsymbol{d}$.

\section{References}

Andrews D L $1976 P h D$ Thesis University of London 1980 J. Phys. B: At. Mol. Phys. 13409

Andrews D L and Ghoul W A $1981 J$. Chem. Phys. (in press)

Andrews D L and Thirunamachandran T 1977 J. Chem. Phys. 675026

Boyle L L and Matthews P S C 1971 Int. J. Quantum Chem. 5381

Caldonazzo B 1932 Rend. Reale Accad. Naz. Lincei 15840

Cayley A 1874 Mon. Not. R. Astron. Soc. 34303

Healy W P 1975 J. Phys. A: Math. Gen. 8 L87

Kearsley E A and Fong J T $1975 J$. Res. NBS 79 B 49

Rivlin R S 1955 J. Rat. Mech. Anal. 4681

Smith G F 1968 Tensor 1979

1972 Tensor 24374

Sokolnikoff I S 1964 Tensor Analysis 2nd edn (New York: Wiley)

Weyl H 1946 The Classical Groups (Princeton: University Press) 